are fundamental in chemotherapy and toxicology, as well as in investigations of enzyme structure, function and mechanism. Both the practical and academic aspects of the subject are well illustrated in this book, which should appeal equally to the enzymologist and the pharmacologist. From the results of kinetic measurements with large numbers of substrates and inhibitors of systematically varied structure, detailed conclusions are drawn about the modes of binding to several enzymes. The value of this type of comparative kinetic investigation of enzymes with wide specificity was shown by the work of Neurath, Niemann and others on chymotrypsin. This work, and the results of similar studies of a number of enzymes concerned in nucleotide metabolism, largely from Professor Baker's own laboratory, are considered here as the first stage in a systematic approach to the design of "active-site-directed irreversible inhibitors" for these enzymes.

This term was coined by Professor Baker to describe a compound with the properties of both a reversible competitive inhibitor and an irreversible inhibitor. This can form a covalent bond with the enzyme by facile neighbouring-group reaction within the reversible complex, thereby making the inhibition irreversible and "labelling", a single group near the active centre. Such a bifunctional inhibitor is likely to be more specific and potent than either of its component types. Di-isopropyl phosphofluoridate was the first to be discovered, and its mechanism and that of azaserine, are considered in the introductory chapter, together with the general principles and potentialities of this kind of inhibition, and some general problems of chemotherapy illustrated by 6-mercaptopurine. In a later chapter, more recent work on other "affinity labelling" reagents for chymotrypsin is discussed, and the rate equations for enzyme inactivation by various mechanisms are derived.

The second chapter is a valuable survey of the types of bonds involved in reversible enzyme-substrate and inhibitor compounds, and the potentialities for such bond formation by protein groups and common functional groups of substrates and inhibitors. Kinetic "binding", studies with chymotrypsin, trypsin, thymidine kinase and phosphorylase, adenylosuccinate synthetase and guanine de-aminase are discussed in detail in succeeding chapters, with the objective of discovering which substrate or inhibitor groups are needed for strong binding, and where a bulky alkylating group, for example, can be tolerated on a good reversible inhibitor.

The last stage in the design of an inhibitor of value in chemotherapy, which is Professor Baker's principal concern, is based on the idea that greater specificity will be achieved if the alkylating group can bridge to a nucleophilic enzyme group outside an active centre, where analogous enzymes from a different species or tissues may differ in structure. The last half of the book is devoted to systematic studies to this end with lactate and glutamate dehydrogenases, tetrahydrofolate dehydrogenase, adenosine de-aminase and thymidilate synthetase. For the first four of these, active-site-directed irreversible inhibitors were discovered; selective specificity for heart and skeletal muscle lactic dehydrogenase was also achieved, but not as yet the main objective of species specificity for the other enzymes. The book closes with a chapter on enzyme-specific columns.

In the face of such a formidable body of kinetic data and such fascinating and detailed rationalizations in terms of binding, it may seem carping to wish for some justification for the interpretation of Michaelis constants and inhibitor constants as dissociation constants, especially for the two-substrate and coenzyme-substrate enzymes studied. Some of the inconsistencies in the rationalizations may well be due to comparisons of $K_{m}$ and $K_{i}$, and to an over-simplified treatment of inhibition. While it may be better to base the design of inhibitors for chemotherapy on doubtful generalizations than to proceed by random synthesis, some of the conclusions about mechanisms may be seriously challenged, and the reader interested in this aspect must be aware of the assumptions underlying the whole approach. Nevertheless, this stimulating and timely book can be recommended to all concerned in any way with enzyme action. There are numerous references to the recent literature, the author's style and presentation are clear, and very few misprints have escaped the proof reader. Consequently, for a book in which some hundreds of compounds are listed in tables and discussed by number in the text, it is remarkably easy to read.

K. Dalziel

\section{MARINE MICROBIOLOGY}

Microbial Population of Oceans and Seas

By A. E. Kriss, I. E. Mishustina, I. N. Mitskevich and E. V. Zemtsova. Translated by K. Syers. Pp. 287. (London: Edward Arnold (Publishers), Ltd., 1967.) $90 s$. Microbial Population of Oceans and Seas by Kriss, Mishustina, Mitskevich and Zemtsova is similar in many respects to Professor Kriss's previous book, Marine Microbiology (Deep Sea), to which it is a supplement. The book itself is primarily an account of the authors' own work describing the species of micro-organisms they have isolated from the sea and the physiological and biochemical properties of their isolates.

The book is divided into three sections. The first section includes useful lists of species of micro-organisms isolated from the sea by various workers, an account of the salt requirement of the authors' isolates, the viability of these organisms, attacks on the work of Sorokin and Kuznetsov, as well as a chapter dealing with the distribution of organic matter in the sea. The latter chapter is in reality an account of distribution of bacterial numbers in the sea, presumed by the authors to be a measure of the amount of labile organic matter. This chapter contains some rather surprising statements about the distribution of organic material in the sea.

The next section is largely devoted to a description of the occurrence and features of some 200 species of bacteria and fungi isolated by the authors from various oceans. The bacteria, comprising the majority of the isolates, are classified by a Russian key. This section also contains a chapter devoted to the occurrence of bacteriocidal properties and bacteriophages among their isolates.

The final section contains short chapters on geographical distribution of bacterial numbers, a genera! discussion of the distribution of bacterial species and a detailed account of the occurrence of one particular species: Bacterium (probably Achromobacter) agile. In addition there is a chapter describing the variation in biochemical activities of species isolated from various localities. The authors place considerable stress on the rosults of this study, and in view of this it is unfortunate that the value of the tests employed is not discussed: for the ability to oxidize lactose or to produce hydrogen sulphide from proteins would not seem to be an important biochemical function of a marine micro-organism. The results of this chapter in conjunction with those of the distribution of organic matter lead the authors to a rather improbable conclusion regarding the cycle of organic material in the ocean and oceanic circulation in general.

The book suffers from a general shallowness of approach as well as containing some rather questionable assumptions and statements. Professor Fogg's editorial comments are in this respect invaluable, directing attention to contentious points. The text contains an enormous amount of information, much of which is unassimilated; consequently the book will be useful to the specialist primarily: it would leave the beginner floundering somewhat.

P. J. Le B. Williams 